American Journal of Pharmaceutical Education 2019; 83 (8) Article 7335.

\title{
RESEARCH
}

\section{Aiding Transformation from Student to Practitioner by Defining Threshold Concepts for the Pharmacists' Patient Care Process}

\author{
Claire Kolar, PharmD, PhD, Kristin K. Janke, PhD \\ Pharmaceutical Care \& Health Systems, University of Minnesota College of Pharmacy, Minneapolis, Minnesota \\ Submitted August 16, 2018; accepted January 28, 2019; published October 2019.
}

Objective. To explore critical milestones in the transition from student to practitioner by identifying the threshold concepts associated with learning the Pharmacists' Patient Care Process (PPCP).

Methods. A threshold concept represents a transformed way of thinking about a topic or discipline. This study convened five focus groups to identify possible threshold concepts related to learning the PPCP. The data were analyzed by deductive content analysis and confirmed by an expert consensus panel using a modified nominal group technique.

Results. Moving beyond the process outlined by the PPCP, the five focus groups identified additional elements of learning required in becoming a patient care practitioner. These elements were focused on the more intangible aspects of patient-centered care, such as practitioner priorities, attitude, and approach. As such, the data help to describe how practitioner's think, feel, and act (ie, their identity). Deductive content analysis resulted in five Patient Care Threshold Concepts (PCTC), which were confirmed by the expert consensus panel.

Conclusion. The five PCTCs provide pharmacy educators with an additional tool to use in teaching the PPCP. The PCTCs can aid in the student-practitioner transformation by helping to make implicit aspects of patient care more explicit. They are supplements to patient care competencies that help explain the practitioner's priorities, attitude, and approach. They can also serve as guidance to pharmacy educators in evaluating curricular activities and assessments, as well as identity formation. This approach can be applied to the identification of threshold concepts in other areas of pharmacy education, such as leadership.

Keywords: threshold concepts, Pharmacists' Patient Care Process, identity, practitioner transformation, student development

\section{INTRODUCTION}

Pharmacy educators have become increasingly interested in exploring the question of how a pharmacy student becomes a practitioner. This question became even more relevant with the development of the Pharmacists' Patient Care Process, ${ }^{1}$ by the Joint Commission of Pharmacy Practitioners (JCPP). The process has five steps (ie, collect, assess, plan, implement, and follow-up) and is intended to be used to inform the way pharmacists provide patient care and to guide pharmacy student instruction. ${ }^{1}$ Before the JCPP's publication of the Pharmacists' Patient Care Process (PPCP), pharmacists and pharmacy educators did not have a standard approach to patient-centered care to be practiced in all settings, which has become more important as pharmacist continue to move into pa-

Corresponding Author: Kristin K. Janke, 7-125D Weaver Densford Hall, 308 Harvard St. SE, University of Minnesota College of Pharmacy, Minneapolis, MN 55455. Tel: 612-6264648. E-mail: janke006@umn.edu tient care roles. ${ }^{1}$ Using a theoretical framework to examine the process of internalizing the PPCP can help pharmacy educators better understand the transformation pharmacy students undergo as they learn to practice patient-centered care.

In 2003, Meyer and Land first defined threshold concepts as "akin to a portal, opening up a new and previously inaccessible way of thinking about something," and representing "a transformed way of understanding, or interpreting, or viewing something without which the learner cannot progress." 2 Identifying threshold concepts is a way for educators to closely examine what is taught, in addition to why and when material is taught. ${ }^{3}$ Threshold concepts provide a way for educators to more deeply understand learning in order to help students master a discipline or anticipate learning struggles.

Meyer and Land identified five defining characteristics of threshold concepts. ${ }^{2}$ First, threshold concepts are transformative. In other words, understanding a threshold concept can lead to any type of transformation 


\section{American Journal of Pharmaceutical Education 2019; 83 (8) Article 7335.}

of the learner, such as a shift in personal identity or values. ${ }^{2}$ They are irreversible, as the learner is likely unable to return to the previous way of thinking after the new perspective is gained. ${ }^{2}$ They are integrative, which "exposes the previously hidden interrelatedness of something." "Threshold concepts are often bounded, meaning the concept interfaces with the edges of a discipline. ${ }^{2}$ Finally, they are troublesome, meaning the learner may struggle with a concept because it is counterintuitive, it comes from an alternative perspective, or it is incoherent. ${ }^{4}$ These five characteristics - transformative, irreversible, integrative, bounded, and troublesomeprovide the framework needed to identify threshold concepts.

Identifying threshold concepts is one method to better understand the way students are transformed into patient-centered care practitioners and to provide insights to influence the teaching of the PPCP. Since the PPCP was published in 2014, publications in pharmacy education literature have focused on specific learning exercises incorporating the $\mathrm{PPCP},{ }^{5}$ integration of the PPCP into a specific course sequence, ${ }^{6}$ and operationalizing the PPCP within a school's curriculum. ${ }^{7}$ Rivkin discussed the relation of the PPCP to pharmacy education more broadly, highlighting the need for early introduction, ${ }^{8}$ and a more recent publication advocated for teaching the PPCP within the framework of pharmaceutical care. ${ }^{9}$ However, there is a need for further discussion and exploration of the milestones in learning the PPCP and the ways students progress in their mastery and internalization of the process. A gap exists between learning the steps of providing patient care as outlined by the PPCP (ie, collect, assess, plan, implement, follow-up) and mastering the complexities of being a pharmacy practitioner. To develop the identity of a practitioner, students must do more than execute the steps of the patient care process; they must learn to think, feel, and act like a practitioner. ${ }^{10}$ This includes learning a practitioner's priorities, attitude, and approach. Threshold concepts can help close that gap. They can also help guide decisions related to teaching and learning patient-centered care. The aim of this study was to explore critical milestones in the transition from student to practitioner by identifying the threshold concepts associated with learning the PPCP.

\section{METHODS}

A qualitative approach was selected to explore student learning from a variety of perspectives and delve into students' and pharmacists' experiences in transitioning to a practitioner. This study used three methodological components to identify and refine threshold concepts. First, focus groups were held to generate data. Second, this data was evaluated using a deductive content analysis approach. Finally, consensus was reached on the findings by employing a modified nominal group technique (NGT) with a panel of experts.

Five focus groups were convened over the course of two months to identify potential threshold concepts. Focus groups were selected because they create conversation and dialogue among participants, ${ }^{11}$ which is advantageous when discussing a relatively intangible subject such as threshold concepts. ${ }^{3}$ In addition, focus groups have been used in other studies identifying threshold concepts. ${ }^{12,13}$ Students, faculty members, residents, and preceptors associated with the University of Minnesota College of Pharmacy (UMN-COP) and with ambulatory care experience were asked to participate in the focus groups. At the UMN-COP, a patient care process has been taught consistently and practiced within ambulatory settings for the past 20 years. As a result, focus group participants from ambulatory care practice had a similar foundation and starting point from which to engage in a conversation about the PPCP.

The first focus group consisted of current ambulatory care residents in the University of Minnesota Postgraduate Pharmacy Residency Program with one to two years' experience providing direct patient care. Twenty-three residents were identified from the program website and invited to participate. The second focus group was made up of faculty members or instructors at the UMN-COP with two or more years' experience teaching the patient care process as defined by Cipolle and colleagues, ${ }^{14}$ which is aligned with the PPCP, ${ }^{1}$ in the classroom or an experiential setting. Twenty-four classroom and experiential faculty members were identified through teaching records and the college website as meeting the criteria and were invited to participate. The third focus group consisted of practicing pharmacists with two or more years' experience teaching UMN-COP students the patient care process at an ambulatory care rotation site. The list of preceptors meeting the criteria was obtained from the Experiential Education Office, and 51 preceptors were invited to participate. The two remaining focus groups were made up of pharmacy students enrolled at the UMN-COP at the time of the study. The fourth focus group consisted of rising second- and third-year students with experience learning the patient care process in the classroom. Course instructors and teaching assistants identified 61 students as potential focus group candidates able to comment on their learning of the patient care process. The fifth focus group was made up of fourth-year students who had completed the ambulatory care advanced pharmacy practice experience (APPE) at the time 


\section{American Journal of Pharmaceutical Education 2019; 83 (8) Article 7335.}

of the study. A list of these students was provided by the Experiential Education Office, and 63 students were invited to participate.

Focus group participants were invited to participate via email. The UMN-COP operates a dual-campus model and has ambulatory care sites across the state. As a result, the focus groups were held online using the Webex videoconferencing system (Cisco, San Jose, CA) to ensure participation was not limited by geography. The focus group questions and Webex technology were piloted with three graduate students to ensure the questions were clear and technology use was feasible and agreeable. The primary investigator led each focus group, and each session was recorded using technology within the Webex platform. A second moderator was present to make written observations during the session. Three graduate students rotated through the role of second moderator and each was instructed to monitor the focus group for technology issues and document anything they heard that stood out to them as worth noting. Each focus group lasted between one to two hours and was followed by a short debrief between the primary investigator and the second moderator.

The goal of the focus groups was to generate a list of potential threshold concepts associated with learning the PPCP, which could be refined and used in later stages of the study. Each focus group answered questions around troublesome concepts, transformative experiences, and pivotal moments experienced when learning to become a patient-centered care practitioner. Questions were designed to elicit ideas, examples, and stories related to the threshold concepts associated with learning the PPCP. The questions were developed by the primary investigator after consulting the literature on threshold concepts $^{2,3}$ and by reviewing a focus group study in which threshold concepts were identified for occupational therapists. ${ }^{13}$

Krueger's method for developing a focus group schedule of questions was followed. ${ }^{15}$ Each focus group started with opening questions to introduce participants and have them start thinking about the topic (eg, "What's the first thing that comes to mind when you think about a student learning the patient care process?"). This was followed by transition questions to get participants to delve deeper into the topic, eg, "Describe a pivotal, or light bulb-type moment you have had observing a student learning or using the patient care process, " as well as key questions which were the main questions informing the analysis, eg, "What is troublesome, or difficult, for students when learning the patient care process?" Finally, the focus group concluded with an ending question to bring closure and provide an opportunity for reflection, eg, "Is there anything you want to share about learning the patient care process that has not come up?"

The data from each focus group was analyzed by the primary investigator using a deductive content analysis approach. ${ }^{16}$ The recordings of each focus group were transcribed. The transcripts were then coded and the codes grouped into categories. The categories were then collapsed into themes. The primary investigator used the threshold concept framework to interpret the data. The question, "Does this describe a concept that could be transformative, irreversible, integrative, bounded, or troublesome to a pharmacy student learning the PPCP?"13 was used as a guide in the deductive approach of identifying codes, categories, and themes from the data. For the purpose of this study, the themes were considered potential threshold concepts. Once the themes, or potential threshold concepts, were identified, the primary investigator wrote a description of the threshold concept using language from the supporting codes and categories.

Once the focus groups were complete and the list of potential threshold concepts with descriptions was generated from the data, an expert consensus panel was convened to verify the analysis of the focus group data. Consensus methods traditionally have been used to "define levels of agreement" on a variety of topics ${ }^{17}$ and are useful in the identification of threshold concepts so they can be used with confidence in curricular decisions. ${ }^{3}$ The goal of the expert consensus panel was to come to agreement on a refined list of threshold concepts from the perspective of teaching the PPCP. The panel was comprised of faculty members involved in teaching the patient care process to pharmacy students. The 24 individuals from the faculty focus group also met the expert criteria of having two or more years' experience teaching the patient care process in a classroom or experiential setting and were invited to participate in the expert consensus panel.

The panel came together for a single workshop designed to build a consensus list of threshold concepts ${ }^{18}$ using a modified nominal group technique (NGT). ${ }^{19}$ The NGT was selected as the consensus method for this study because it involves face-to-face interaction between participants and is easily able to be modified for specific situations. The traditional NGT is a four-step consensus process consisting of participants generating ideas, sharing ideas, discussing the ideas, and voting on the ideas. Other researchers have recommended that an NGT workshop have no more than seven participants, be led by a moderator, and consist of experts with knowledge about the ideas or topics being discussed. ${ }^{19}$

In the present study, the modified NGT used the list of potential threshold concepts previously identified from the analysis of the focus group data as the starting point for 


\section{American Journal of Pharmaceutical Education 2019; 83 (8) Article 7335.}

the process rather than having participants generate a list of ideas for discussion and voting. For a threshold concept to be included in the final consensus list, it needed to be transformative and have one other defining characteristic of threshold concepts (ie, irreversible, integrative, bounded, or troublesome).$^{13}$ Other researchers have recommended that consensus be defined prior to the NGT session, ${ }^{17}$ but a set level of agreement does not exist in the literature. A simple majority, ${ }^{20}$ two-thirds majority, ${ }^{17}$ or $80 \%$ agreement ${ }^{20}$ could all be considered as achieving consensus depending on the type of consensus method used and the needs of the study. In this study, a threshold concept was included in the final list when two-thirds (67\%) of the experts determined it met the defined criteria.

Prior to the panel convening, experts were sent background materials to review regarding threshold concepts. Using videoconferencing, the panel came together for a 90-minute discussion of the list of potential patient care threshold concepts and consensus voting. The session started with a conversation of threshold concepts, in general, followed by a discussion of each of the potential patient care threshold concepts. Experts read the first threshold concept and its short description and had a few minutes on their own to make notes on their impression of the threshold concept or questions they had about it. Then, the panel discussed the degree to which the proposed threshold concept aligned with the definition of a threshold concept and which of the five characteristics were met. This process was repeated for the remaining potential patient care threshold concepts. After each concept had been discussed as a group, experts voted individually using paper ballots on whether they thought the proposed threshold concept met the criteria and which of the five characteristics were applicable. The votes were collected and tabulated by the primary investigator after the session ended. Revisions were made to the language of two of the potential patient care threshold concepts by the primary investigator to provide clarifications raised by the panel. The revised threshold concepts were sent back to the experts via email for a second round of electronic voting. Partici- pants had one week to review the update threshold concepts and return the ballots via email. This study was determined to be exempt from review by the University of Minnesota Institutional Review Board.

\section{RESULTS}

In total, five focus groups were held to identify threshold concepts associated with learning the PPCP (Table 1). The coding of the focus groups transcripts resulted in 14 initial categories (Table 2). The categories were labeled and supporting codes from all five focus group transcripts were added to the corresponding category to provide additional detail and context to be used for the next round of analysis. After all the categories and supporting codes were reviewed by the primary investigator, one was discarded (category 9, "Reflection is needed. ..") because it was determined not to be a potential threshold concept but instead a strategy for learning the PPCP. From the remaining 13 categories, five themes ie, threshold concepts, emerged from the data. The themes were written as threshold concept statements, and each was supported by a detailed description based on the categories and codes (Table 3 ).

Six faculty members volunteered and were available to participate in the expert consensus panel. One expert primarily taught the patient care process in the classroom, two taught patient care in a skills laboratory course, and five out of the six served as preceptors in ambulatory care settings. One expert had previously participated in the faculty focus group. The panel came to consensus on three of the five threshold concepts, based on their initial responses on the paper ballots. At least two-thirds of the panel agreed that PCTCs 1, 2, and 4 met the definition of threshold concepts, were transformative, and included at least one other defining characteristic. However, consensus was not reached regarding PCTCs 3 and 5.

Based on the discussion during the NGT session and comments made by the experts on their ballots, the primary investigator reviewed PCTCs 3 and 5, the categories which informed these threshold concepts, and the original codes supporting the categories. While agreeing PCTC 3

Table 1. Demographics of Focus Groups Conducted to Identify Threshold Concepts Associated with Learning the Pharmacists' Patient Care Process

\begin{tabular}{|c|c|c|c|c|}
\hline Focus Group & Participants & Invited, No. & Participated, No. & Length \\
\hline 1 & Ambulatory Care Residents & 23 & 10 & $1 \mathrm{hr}, 48 \mathrm{~min}$ \\
\hline 2 & Second and Third Year Student Pharmacists & 61 & 9 & $1 \mathrm{hr}, 50 \mathrm{~min}$ \\
\hline 4 & Fourth Year Student Pharmacists & 63 & 6 & $1 \mathrm{hr}, 22 \mathrm{~min}$ \\
\hline 5 & Ambulatory Care Pharmacy Faculty & 24 & 8 & $1 \mathrm{hr}, 57 \mathrm{~min}$ \\
\hline
\end{tabular}




\section{American Journal of Pharmaceutical Education 2019; 83 (8) Article 7335.}

Table 2. Categories Identified From Deductive Content Analysis of Input From Focus Group Members in a Study to Identify the Threshold Concepts Associated with Learning the Pharmacists' Patient Care Process

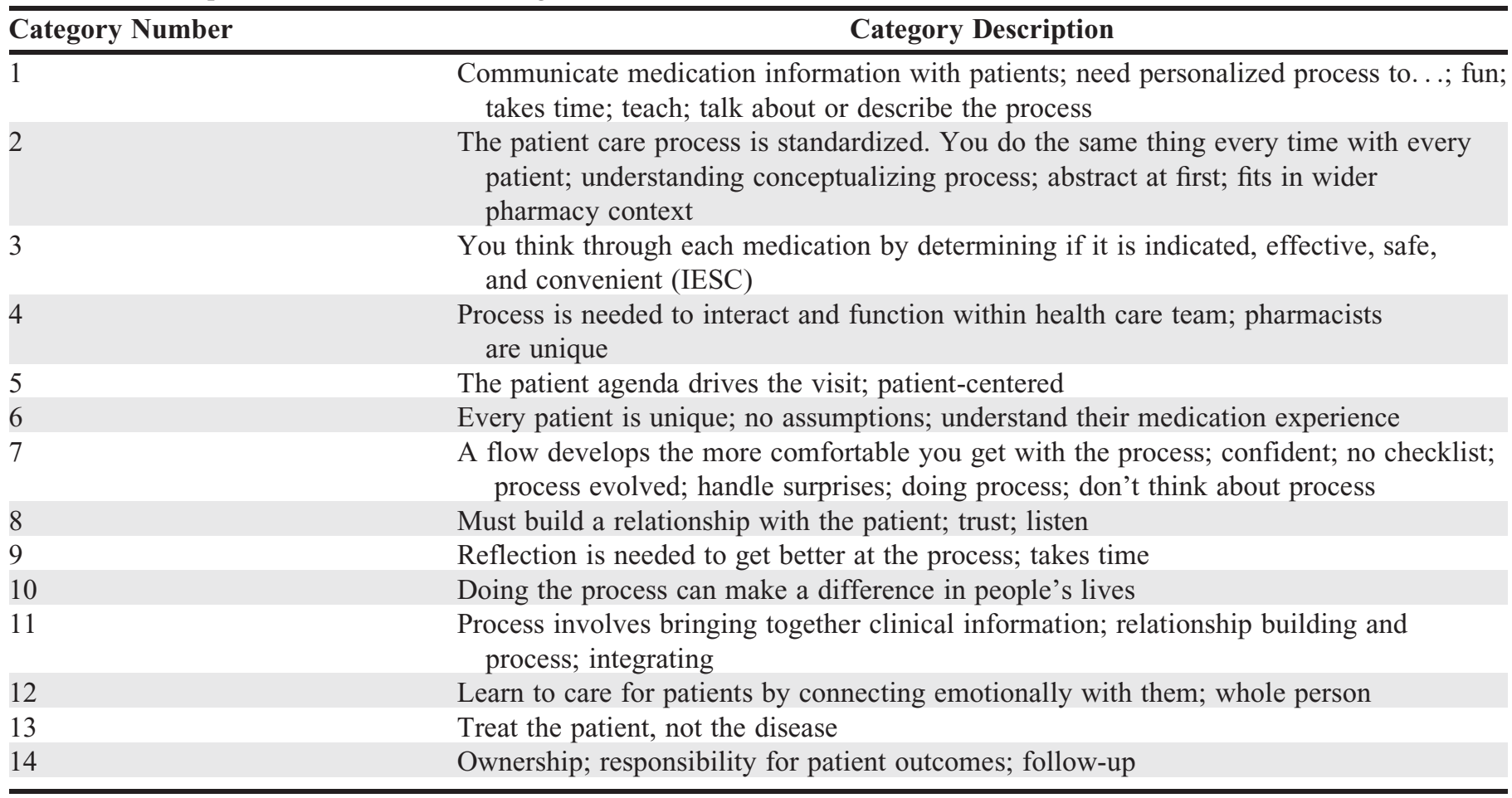

was a threshold concept, the experts felt the initial wording of the threshold concept statement did not accurately reflect the description. After revisiting the data, the primary investigator reworded the threshold concept to better align with the supporting categories and codes (Table 3 ). As written, the panelists determined PCTC 5 was not a threshold concept. Instead, it seemed like a description of a learner's approach to patient care once the threshold had been crossed. Again, after revisiting the data, the primary investigator determined this theme did emerge from the data as a threshold concept and this statement was also revised to better reflect the supporting categories and codes (Table 3). The descriptions for PCTC 3 and 5 were not changed.

The revised PCTCs were sent to the experts via email for a second round of consensus voting. At least twothirds of the experts came to consensus regarding revised PCTCs $3(67 \%)$ and 5 (83\%). The final five patient-care threshold concepts are provided in Table 3, with labels and descriptions.

\section{DISCUSSION}

The study identified and defined threshold concepts to further pharmacy educators' understanding of the transformation that occurs as pharmacy students become patient care practitioners. These five threshold concepts capture aspects of patient care that are integral to learning but can be difficult to name or define. These threshold concepts illustrate various aspects of the transformation that students undergo as they become practitioners and can be used to bring about that transformation in various ways within pharmacy education.

The patient care threshold concepts (PCTCs) can be considered in designing specific learning activities to create specific opportunities for students to transform in specific ways. For instance, a patient care encounter could be designed to incorporate PCTC 4, "Discern a patient's medication experience and incorporate his or her individual knowledge and beliefs into the care provided (Medication Experience)." Regardless of setting, students should be trained to inquire about a patient's understanding of their medication, their view of their medications, and any values or beliefs impacting their medication-taking behavior. ${ }^{14}$ Students should learn that a medication history is not complete without the medication experience of the patient. ${ }^{14}$ Exercises and assessments can be designed to ensure that the student is adept at eliciting, hearing, considering, and responding to elements of a patient's medication experience.

With the publication of the PPCP, a consistent patient care process was created that made many aspects of patient-centered care explicit. The publication of the Common Language Document from the American College of Clinical Pharmacy further defined elements of 


\section{American Journal of Pharmaceutical Education 2019; 83 (8) Article 7335.}

Table 3. Patient Care Threshold Concepts and Descriptions Associated with Learning the Pharmacists' Patient Care Process

Patient Care Threshold Concept 1: Provide care in which the patient is at the center of each decision made throughout the process [Patient Centeredness] ${ }^{\mathrm{a}}$

Pharmacists provide patient-centered care by listening to patients and putting their needs, concerns, and desires ahead of their own agenda for the encounter or what should be the outcome, based on guidelines or a preconceived plan. Pharmacists see patients as a whole person, not a series of disease states or drug therapy problems.

Patient Care Threshold Concept 2: Conceptualize and articulate pharmacists' unique patient care process [Unique Process] ${ }^{\mathrm{a}}$ Pharmacists have a standardized process, using common language (including Indication, Effective, Safe, Convenient, or IESC) and integrating clinical knowledge to provide patient care in a way that is distinct, yet complementary to other health professions. The process is universal and can be followed by any pharmacist in any setting to solve problems arising when providing patient care.

Patient Care Threshold Concept 3: Create an impact on care by establishing and building a relationship with the patient and taking responsibility for their outcomes [Relationship and Responsibility] ${ }^{\mathrm{a}}$

Pharmacists have an impact on a patient's care when they develop a relationship with the patient and connect emotionally with them. Pharmacists can make a difference in a patient's life as they work together over time and take ownership of the patient's care and outcomes. ${ }^{\mathrm{b}}$

Patient Care Threshold Concept 4: Discern a patient's medication experience and incorporate his or her individual knowledge and beliefs into the care provided [Medication Experience] ${ }^{\mathrm{a}}$

Pharmacists recognize each patient has a unique view of their medications and distinct medication taking behavior. Regardless of how one appears on paper, pharmacists do not make assumptions about patients' understanding of medications or their expectations of care. Pharmacists work with patients to uncover the individual complexities of their life and their goals related to medications and use this information when providing care.

Patient Care Threshold Concept 5: Internalize the patient care process as an automatic or ingrained approach to patient care, adapted to each patient, rather than a series of discreet steps [Ingrained Approach] ${ }^{\mathrm{a}}$

Pharmacists are able to provide effective, individualized patient care when they internalize the patient care process, use it routinely without actively thinking about it, enhance it with their own style, and adapt it to respond to the patient in front of them. ${ }^{\mathrm{c}}$

${ }^{a}$ Bracketed text refers to the label for each concept

${ }^{\mathrm{b}}$ Initial wording: Establish and continually build a relationship with the patient

${ }^{c}$ Initial wording: Internalize the patient care process in order to provide care

the process. ${ }^{21}$ However, other important facets of providing care are more implicit, or mentioned but not fully drawn out. The PCTCs can be viewed as complementary to the PPCP and can make some of the implicit components of providing care more explicit. For example, PCTC 5, "Internalize the patient care process as an automatic or ingrained approach to patient care, adapted to each patient, rather than a series of discreet steps (Ingrained Approach)," is an illustrative and specific statement about the process of providing patient-centered care. Patient care threshold concept 5 describes the need to internalize and practice the process as a whole rather than as isolated steps, and therefore complements and enriches the teaching of the PPCP. Concept 5 also emphasizes routine use, the degree of fluency with the process (ie, automatic, without actively thinking about it), adaption to allow for the individualized nature of care, and enhancement with the practitioner's own style.

The PCTCs can also be used to orient a pharmacy student to their role. They begin to define elements of "thinking, feeling, and acting" like a practitioner, such as the practitioner's priorities, attitudes, and approach. In particular, work related to PCTC 2, "Conceptualize and articulate pharmacists' unique patient care process (Unique Process)," can help a student pharmacist develop his or her own professional identity, including their identity in relation to other health professionals. As students advocate for patients and their care with other providers, they need to possess a solid professional identity. Furthermore, students must be able to understand and articulate their unique role in the patient's care. The development of a professional identity is becoming an important aspect of professional education. In "Educating Physicians: A Call for Reform of Medical School and Residency," Cooke and colleagues summarized a Carnegie Foundation medical education study and recommended four goals, the fourth being a "focus on professional identity formation." ${ }^{22}$ In 2015, Mylrea and colleagues reviewed the state of professional identity development in pharmacy education and concluded that the profession needs to "move beyond the demonstration of desirable behaviors, attitudes, and values to a more holistic approach of professional identity formation." 23 By considering the PCTCs, educators can be better attuned to the transitions occurring and better target 


\section{American Journal of Pharmaceutical Education 2019; 83 (8) Article 7335.}

learning activities that move beyond skill development and aid in identify formation.

Threshold concepts, by design, are related to both cognitive and affective domains; they shift how a learner views something intellectually and also how he or she experiences it. ${ }^{24}$ As such, they may be an asset in supplementing existing competency statements and assessment tools. For example, Entrustable Professional Activities (EPAs) have developed as a way to assess practice skills and typically require multiple competencies to demonstrate the standard has been met. ${ }^{25}$ The PCTCs can be used to refine and enrich a patient-centered care EPA, to draw out nuances for learners, and to give educators additional insight into ways an EPA can be achieved. For example, a patient care EPA can be supplemented by intentionally incorporating and emphasizing PCTC 3 , "Create an impact on care by establishing and building a relationship with the patient and taking responsibility for their outcomes (Relationship and Responsibility)." This might include debriefing on challenges with relationship building after a care encounter or preceptor feedback on the student's areas for improvement in accepting responsibility. The learner may gain insight into the way experienced practitioners interact with patients, and the evaluator may now have language to describe something lacking from a learner's approach to patient care.

By examining and articulating milestones in learning the PPCP, this study illustrates that a transformation is going on below the surface as students become practitioners. Threshold concepts, by definition, are transformative $^{26}$ and they identify and describe areas that require transformative learning experiences. In transformative learning theory, Meizrow posits that learners undergo shifts in their frames of reference that result in new perspectives. ${ }^{27}$ More work is needed to examine the development of transformative learning experiences that may assist in creating movement over these patient care thresholds. In addition, threshold concepts may be a useful lens through which to evaluate the curriculum. An analysis could be undertaken to determine when specific threshold concepts are addressed and when they are fully met, along with the teaching/learning methods used. This evaluative data may yield ideas for improvement of the student learning experience.

The identification of threshold concepts in pharmacy education does not need to be limited to the PPCP. Patient-centered care provider, or caregiver, is only one of the 15 educational outcomes outlined by the Center for the Advancement of Pharmacy Education (CAPE) in 2013. ${ }^{28}$ Pharmacy graduates are also expected to be problemsolvers, educators, and health advocates, for example. Because of their role in linking together the cognitive and affective domain, the identification of threshold concepts may be particularly relevant to the CAPE outcomes in the personal and professional development domain, such as leadership.

The approach used to identify threshold concepts in this study had several strengths, including incorporation of viewpoints from various development stages (ie, those in transition, those through the transition) and a collaboration between students, academics, and those in the professional community. ${ }^{3}$ In addition, the wide range of participants (students, residents, faculty members, and preceptors) was intentionally sought out to increase transferability of findings to other patient care and educational settings, which is one goal of qualitative research. Fidelity to the threshold concepts theory was aided through the use of deductive analysis based on the defining characteristics of threshold concepts ${ }^{13}$ and by panelist voting based on the same characteristics. Also, the use of sequential and varied data collection allowed for checking the data, refining the statements, and affirming the findings. In particular, the use of a nominal group process allowed a variation of member checking, while also allowing consensus to be obtained. Finally, incorporating consensus methodology aided in threshold concept identification by promoting discussion around the identified threshold concepts and increasing their applicability in educational settings once consensus was reached. ${ }^{3}$

This study has some limitations. This research specifically sought focus-group participants with patient care experience in ambulatory care settings. The PPCP is intended to be used regardless of setting, so additional research may be needed to verify these results in other practice settings. In addition, the study was conducted at a single institution. Although all focus group participants and expert consensus panelists were affiliated with a single institution, they had a variety of educational experiences (ie, not all were alumni, some residency trained/ some not, different residencies) and professional experiences (ie, different practices and years in practice). Additional research with participants from additional curricula and additional institutions with varying commitments to the PPCP, history with the PPCP, and practice environments is needed. Finally, while a deductive approach to analysis ensures the findings align with the threshold concept framework, it might limit the type or number of themes emerging from the data.

\section{CONCLUSION}

This study aimed to explore critical milestones in the transition from student to practitioner by identifying the threshold concepts associated with learning the pharmacists' patient care process by drawing on the specific 


\section{American Journal of Pharmaceutical Education 2019; 83 (8) Article 7335.}

experience and expertise of students, faculty, residents, and preceptors affiliated with ambulatory care practice. Five patient care threshold concepts were identified using focus groups, deductive content analysis, and an expert consensus panel employing a modified nominal group technique. These five PCTCs can be used as a tool in teaching patient care, assessing students, and evaluating curricular activities. They can also aid in the transformation of students into practitioners and contribute to identity development. Incorporating the PCTCs into patient care teaching can supplement existing competencies around practitioner priorities, attitude, and approach, and guide learners in uncovering and defining the way they think, feel, or act when providing patient care. The identification of PCTCs is a key step in helping educators more deeply understand student learning of the PPCP. Finally, this process of threshold concept identification can be applied to other areas of transformation in pharmacy curricula, such as leadership.

\section{ACKNOWLEDGMENTS}

Drs. Ronald Hadsall, Don Uden, and Jane Miller provided input on the study methods and the process of analysis, as well as early versions of this report.

\section{REFERENCES}

1. Joint Commission of Pharmacy Practitioners. Pharmacists' Patient Care Process. https://jcpp.net/wp-content/uploads/2016/03/

PatientCareProcess-with-supporting-organizations.pdf. Published May 29, 2014. Accessed October 8, 2019.

2. Meyer JHF, Land R. Threshold Concepts and Troublesome Knowledge: Linkages to Ways of Thinking and Practising within the Disciplines. Enhancing Teaching-Learning Environments in Undergraduate Courses Project. Occasional Report 4. http:// www.etl.tla.ed.ac.uk/docs/ETLreport4.pdf. Accessed October 8, 2019. 3. Barradell S. The identification of threshold concepts: a review of theoretical complexities and methodological challenges. High Educ. 2013;65(2):265-276.

4. Lucas U, Mladenovic R. The potential of threshold concepts: an emerging framework for educational research and practice. London Rev Educ. 2007;5(3):237-248.

5. Bourg Rebitch C, Fleming VH, Palmer R, Rong H, Choi I.

Evaluation of video-enhanced case-based activities guided by the pharmacists' patient care process. Am J Pharm Educ.

2019;83(4):Article 6676.

6. Gonyeau MJ, DiVall M, Conley MP, Lancaster J. Integration of the pharmacists' patient care process (PPCP) into a comprehensive disease management course series. Am J Pharm Educ.

2018;82(6):Article 6311.

7. Cooley J, Lee J. An operationalization of the Pharmacists' Patient Care Process at on public college of pharmacy. Am J Pharm Educ. 2018;82(2):Article 6301.

8. Rivkin A. Thinking clinically from the beginning: early introduction of the pharmacists' patient care process. Am J Pharm Educ. 2016;80(10):Article 164.
9. Kolar C, Hager KD, Losinski V. Beyond the pharmacists' patient care process: cultivating patient care practitioners by utilizing the pharmaceutical care framework. Inov Pharm. 2017;8(3):Article 7. 10. Cruess RL, Cruess SR, Steinert Y. Amending Miller's pyramid to include professional identity formation. Acad Med. 2016;91(2):180185.

11. Russ-Eft D, Preskill H. Evaluation in Organizations: $A$ Systematic Approach to Enhancing Learning, Performace, and Change. 2nd ed. New York, NY: Basic Books; 2009.

12. Loertscher J, Green D, Lewis JE, Lin S, Minderhout V. Identification of threshold concepts for biochemistry. CBE Life Sci Educ. 2014;13(3):516-528.

13. Tanner B. Threshold concepts in practice education: perceptions of practice educators. Br J Occup Ther. 2011;74(9):427-434.

14. Cipolle RJ, Strand LM, Morley PC. Pharmaceutical Care Practice: The Clinician's Guide. 3rd ed. New York, NY: McGraw Hill; 2012.

15. Krueger RA, Casey MA. Focus Groups: A Practical Guide for Applied Research. 3rd ed. Thousand Oaks, CA: Sage Publications, Inc; 2000.

16. Thomas DR. A general inductive approach for analyzing qualitative evaluation data. Am J Eval. 2006;27(2):237-246.

17. Fink A, Kosecoff J, Chassin M, Brook RH. Consensus methods: characteristics and guidelines for use. Am J Public Health. 1984;74(9):979-983.

18. Hesterman DC, Male SA, Baillie CA. Some potential underlying threshold concepts in engineering dynamics. In: Proceedings of the 2011 AAEE Conference, Fremantle, Western Australia; 2011.

19. McMillan SS, King M, Tully MP. How to use the nominal group and Delphi techniques. Int J Clin Pharm. 2016;38(3):655662.

20. Nair R, Aggarwal R, Khanna D. Methods of formal consensus in classification/diagnostic criteria and guideline development. Semin Arthritis Rheum. 2011;41(2):95-105.

21. CMM in Primary Care Research Team. The Patient Care Process for Delivering Comprehensive Medication Management (CMM): Optimizing Medication Use in Patient-Centered, Team-Based Care Settings. 2018. https://www.accp.com/docs/positions/misc/

CMM_Care_Process.pdf. Accessed October 8, 2019.

22. Cooke M, Irby DM, O'Brien BC. Educating Physicians: A Call for Reform to Medical School and Residency. San Francisco, CA: Jossey-Bass; 2010.

23. Mylrea MF, Sen GT, Glass BD. Professionalization in pharmacy education as a matter of identity. Am J Pharm Educ.

2015;79(9):Article 142.

24. Rattray J. Affective dimensions of liminality. In: Land R, Meyer JHF, Flanagan M, eds. Threshold Concepts in Practice. Rotterdam, The Netherlands: Sense Publishers; 2016.

25. ten Cate O, Chen HC, Hoff RG, Peters H, Bok H, van der Schaaf M. Curriculum development for the workplace using Entrustable Professional Activities (EPAs): AMEE Guide No. 99. Med Teach. 2015;37(11):983-1002.

26. Land R, Meyer JHF, Flanagan MT, eds. Threshold Concepts in Practice. Rotterdam, The Netherlands: Sense Publishers; 2016.

27. Mezirow J. An overview of transformative learning. In: Illeris K, ed. Contemporary Theories of Learning. New York, NY: Routledge; 2009:235.

28. Medina MS, Plaza CM, Stowe CD, et al. Center for the advancement of pharmacy education educational outcomes 2013. Am J Pharm Educ. 2013;77(8):Article 162. 